Supplement of Earth Syst. Sci. Data, 11, 111-117, 2019

https://doi.org/10.5194/essd-11-111-2019-supplement

(C) Author(s) 2019. This work is distributed under

the Creative Commons Attribution 4.0 License.

(c) (i)

Supplement of

\title{
Lake O'Hara alpine hydrological observatory: hydrological and meteorological dataset, 2004-2017
}

\author{
Jesse He and Masaki Hayashi \\ Correspondence to: Jesse He (jehe@ucalgary.ca)
}

The copyright of individual parts of the supplement might differ from the CC BY 4.0 License. 
Table S1. Ranges of dates where OHAWS was affected by snow capping (i.e. periods over which precipitation data at OHAWS were approximated from their relationship to precipitation recorded at OPAWS). Total precipitation approximated over snow capping periods is also shown.

\begin{tabular}{|l|l|r|}
\hline Start & End & Total precipitation $(\mathrm{mm})$ \\
\hline $2011-02-14$ & $2011-03-09$ & 37.8 \\
\hline $2012-03-04$ & $2012-04-10$ & 170.0 \\
\hline $2012-12-11$ & $2013-01-15$ & 47.8 \\
\hline $2014-01-09$ & $2014-01-26$ & 41.1 \\
\hline $2014-11-21$ & $2015-01-21$ & 137.6 \\
\hline $2016-11-27$ & $2017-01-11$ & 52.7 \\
\hline
\end{tabular}


Table S2. Summary table listing time series, measurement interval and years of availability. For years highlighted in green, timeseries data are available with no large data gaps ( $>1$ month). For years highlighted in orange, timeseries data are available with one or more large gaps. For years highlighted in white, no timeseries data are available.

\begin{tabular}{|c|c|c|c|c|c|c|c|c|c|c|c|c|c|c|c|c|}
\hline Data set & Time series/Location & Interval & ঠ্ণ & ஜ & ஓ̊ & 옹 & 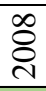 & ஓి & $\stackrel{\circ}{\circ}$ & $\overline{\bar{\nu}}$ & $\frac{\mathrm{N}}{\mathrm{N}}$ & $\stackrel{m}{\circ}$ & 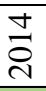 & $\frac{n}{8}$ & $\frac{0}{0}$ & 공 \\
\hline \multirow{4}{*}{ Lake level } & Lake O'Hara raw level & $30 / 60 \mathrm{~min}$ & & & & & & & & & & & & & & \\
\hline & Lake O'Hara corrected level & $30 / 60 \mathrm{~min}$ & & & & & & & & & & & & & & \\
\hline & Opabin Lake raw level & $30 / 60 \mathrm{~min}$ & & & & & & & & & & & & & & \\
\hline & Opabin Lake corrected level & $30 / 60 \mathrm{~min}$ & & & & & & & & & & & & & & \\
\hline \multirow{2}{*}{ Snow survey } & OPAWS & Yearly & & & & & & & & & & & & & & \\
\hline & OHAWS & Yearly & & & & & & & & & & & & & & \\
\hline \multirow{9}{*}{ Stream Discharge } & Gorge & $30 / 60 \mathrm{~min}$ & & & & & & & & & & & & & & \\
\hline & Hungabee & $30 / 60 \mathrm{~min}$ & & & & & & & & & & & & & & \\
\hline & Lefroy Oesa & $30 \mathrm{~min}$ & & & & & & & & & & & & & & \\
\hline & Mary & $30 / 60 \mathrm{~min}$ & & & & & & & & & & & & & & \\
\hline & Oesa Falls & $30 / 60 \mathrm{~min}$ & & & & & & & & & & & & & & \\
\hline & Opabin East & $30 / 60 \mathrm{~min}$ & & & & & & & & & & & & & & \\
\hline & Opabin West & $30 / 60 \mathrm{~min}$ & & & & & & & & & & & & & & \\
\hline & Outlet & $30 / 60 \mathrm{~min}$ & & & & & & & & & & & & & & \\
\hline & Upper Opabin Creek & $30 / 60 \mathrm{~min}$ & & & & & & & & & & & & & & \\
\hline Water chemistry & Various sample locations & $1 / 2$ weeks & & & & & & & & & & & & & & \\
\hline \multirow{26}{*}{ Weather } & OHAWS air temperature & $60 \mathrm{~min}$ & & & & & & & & & & & & & & \\
\hline & OHAWS relative humidity & $60 \mathrm{~min}$ & & & & & & & & & & & & & & \\
\hline & OHAWS wind speed & $60 \mathrm{~min}$ & & & & & & & & & & & & & & \\
\hline & OHAWS wind direction & $60 \mathrm{~min}$ & & & & & & & & & & & & & & \\
\hline & OHAWS raw precip. geonor & $60 \mathrm{~min}$ & & & & & & & & & & & & & & \\
\hline & OHAWS precip. TBRG & $60 \mathrm{~min}$ & & & & & & & & & & & & & & \\
\hline & OHAWS net radiation & $60 \mathrm{~min}$ & & & & & & & & & & & & & & \\
\hline & OHAWS depth to snow & $60 \mathrm{~min}$ & & & & & & & & & & & & & & \\
\hline & OHAWS snow depth & $60 \mathrm{~min}$ & & & & & & & & & & & & & & \\
\hline & OHAWS battery voltage & $60 \mathrm{~min}$ & & & & & & & & & & & & & & \\
\hline & OHAWS corrected precip. & $60 \mathrm{~min}$ & & & & & & & & & & & & & & \\
\hline & OPAWS air temperature & $30 \mathrm{~min}$ & & & & & & & & & & & & & & \\
\hline & OPAWS relative humidity & $30 \mathrm{~min}$ & & & & & & & & & & & & & & \\
\hline & OPAWS wind speed & $30 \mathrm{~min}$ & & & & & & & & & & & & & & \\
\hline & OPAWS wind direction & $30 \mathrm{~min}$ & & & & & & & & & & & & & & \\
\hline & OPAWS raw precip. geonor & $30 \mathrm{~min}$ & & & & & & & & & & & & & & \\
\hline & OPAWS precip. TBRG & $30 \mathrm{~min}$ & & & & & & & & & & & & & & \\
\hline & OPAWS depth to snow & $30 \mathrm{~min}$ & & & & & & & & & & & & & & \\
\hline & OPAWS snow depth & $30 \mathrm{~min}$ & & & & & & & & & & & & & & \\
\hline & OPAWS SW in & $30 \mathrm{~min}$ & & & & & & & & & & & & & & \\
\hline & OPAWS SW out & $30 \mathrm{~min}$ & & & & & & & & & & & & & & \\
\hline & OPAWS LW in & $30 \mathrm{~min}$ & & & & & & & & & & & & & & \\
\hline & OPAWS LW out & $30 \mathrm{~min}$ & & & & & & & & & & & & & & \\
\hline & OPAWS net radiation & $30 \mathrm{~min}$ & & & & & & & & & & & & & & \\
\hline & OPAWS battery voltage & $30 \mathrm{~min}$ & & & & & & & & & & & & & & \\
\hline & OPAWS corrected precip. & $30 \mathrm{~min}$ & & & & & & & & & & & & & & \\
\hline
\end{tabular}


Table S3. Universal Transverse Mercator coordinates of weather (OHAWS and OPAWS) and stream gauging stations (Zone 11, North American Datum 1983).

\begin{tabular}{|l|l|l|}
\hline Station & Easting & Northing \\
\hline OHAWS & 545820 & 5689974 \\
\hline OPAWS & 547234 & 5688222 \\
\hline Gorge & 546951 & 5688770 \\
\hline Mary & 546407 & 5689381 \\
\hline Oesa Falls & 546986 & 5689650 \\
\hline Outlet & 546024 & 5689869 \\
\hline Upper Opabin Creek & 547193 & 5688116 \\
\hline Hungabee & 547340 & 5688082 \\
\hline Opabin West & 546750 & 5689402 \\
\hline Opabin East & 546900 & 5689437 \\
\hline Lefroy Oesa & 547951 & 5689574 \\
\hline
\end{tabular}


Table S4. Measured sensor heights and height adjustments at OPAWS and OHAWS over the monitoring period of $2004-2017$. Heights were measured from sensor to ground surface with measuring tape and are precise up to (roughly) the nearest centimeter. On dates where height measurement corresponds with height adjustment, heights prior to adjustment are given. This is not a complete record of sensor height changes.

\begin{tabular}{|c|c|c|c|c|}
\hline Station & Date & Sensor & Height (m) & Height adjustment \\
\hline \multirow{13}{*}{ OPAWS } & 11-Jun-15 & SR-50 & 2.42 & \\
\hline & & HMP35 & 2.53 & \\
\hline & & Geonor T200B & 2.37 & \\
\hline & & CNR1 & 2.69 & \\
\hline & 28-Aug-12 & SR-50 & 2.49 & \\
\hline & & CNR1 & 2.74 & Raised from $2.51 \mathrm{~m}$ \\
\hline & & HMP35 & 2.60 & Lowered from $3.24 \mathrm{~m}$ \\
\hline & & RM Young 05103 & 4.05 & Lowered from $4.37 \mathrm{~m}$ \\
\hline & 24-Sep-07 & SR-50 & 2.87 & \\
\hline & & CNR1 & 2.71 & \\
\hline & & HMP35 & 3.40 & \\
\hline & & RM Young 05103 & 4.67 & \\
\hline & 20-Jun-06 & SR-50 & 1.56 & \\
\hline \multirow{7}{*}{ OHAWS } & 11-Jun-15 & SR-50 & 2.15 & \\
\hline & & HMP35 & 2.19 & \\
\hline & & NR-Lite & 2.00 & \\
\hline & & RM Young 05103 & 3.10 & \\
\hline & & Geonor T200B & 2.22 & \\
\hline & 28-Sep-11 & SR-50 & 2.10 & \\
\hline & 21-Jun-06 & SR-50 & 1.56 & \\
\hline
\end{tabular}

